\title{
n-butanol extract from Folium isatidis inhibits the lipopolysaccharide-induced downregulation of CXCR1 and CXCR2 on human neutrophils
}

\author{
BEIBEI WU ${ }^{1 *}$, LIYIN WANG $^{2 *}$, LILI JIANG ${ }^{1}$, LILI DONG $^{1}$, FENGLI XU ${ }^{1}$, YILI LU $^{1}$, \\ JIAHUI JIN $^{1}$, ZHANYUE WANG ${ }^{3}$, GUANG LIANG ${ }^{3}$ and XIAOOU SHAN ${ }^{1}$
}

\begin{abstract}
${ }^{1}$ Department of Pediatrics, The Second Affiliated Hospital and Yuying Children's Hospital of Wenzhou Medical University, Wenzhou, Zhejiang 325027; ${ }^{2}$ College of Basic Medical Sciences, Capital Medical University, Beijing 100050; ${ }^{3}$ Chemical

Biology Research Center, School of Pharmacy, Wenzhou Medical University, Wenzhou, Zhejiang 325035, P.R. China
\end{abstract}

Received June 20, 2016; Accepted July 20, 2017

DOI: $10.3892 / \mathrm{mmr} .2017 .7870$

\begin{abstract}
Neutrophils, immune cells crucial for protecting against invading pathogens, are important in sepsis. Neutrophil migration is regulated by chemokine receptors and their cognate ligands. Our previous study investigated the effect of n-butanol extract from Folium isatidis on lipopolysaccharide (LPS)-induced septic shock. The present study stimulated neutrophils with LPS to explore the influence of LPS on cell. Neutrophils were then pretreated with $n$-butanol extract from Folium isatidis followed by LPS to examine the effect of this extract on neutrophil chemotaxis. The results showed that LPS decreased the expression levels of CXC-chemokine receptor (CXCR)1, CXCR2 and L-selectin (CD62L), and increased the expression of interleukin-8 (IL-8) by neutrophils. The addition of $n$-butanol extract from Folium isatidis inhibited this LPS-induced downregulation of CXCR1, CXCR2 and CD62L, and decreased the expression of IL-8 on neutrophils. In addition, $n$-butanol extract promoted myeloperoxidase activity in neutrophils. Taken together, LPS downregulated the expression of chemokine receptors, leading to the failure of neutrophils to migrate to sites of infection. The addition of $n$-butanol extract, which promoted the ability of neutrophils to migrate, is a natural product and potential therapeutic agent with which to target neutrophil chemotaxis during LPS stimulation.
\end{abstract}

Correspondence to: Dr Xiaoou Shan, Department of Pediatrics, The Second Affiliated Hospital and Yuying Children's Hospital of Wenzhou Medical University, 109 West Xueyuan Road, Wenzhou, Zhejiang 325027, P.R. China

E-mail: sssxooo@sina.com

*Contributed equally

Key words: Folium isatidis, lipopolysaccharide, CXC-chemokine receptor 1, CXC-chemokine receptor 2, neutrophil migration

\section{Introduction}

Sepsis is a systemic inflammatory response syndrome associated with a high rate of mortality (1). The incidence of sepsis has been increasing over the last two decades. This increase has been more marked in developed countries, where $6-30 \%$ of patients in Intensive Care Units suffer from sepsis (2). Sepsis remains the leading cause of mortality in patients in Intensive Care Units (3) and the associated burden of care incurs financial burden. However, the pathogenesis of sepsis remains to be fully delineated and effective therapies are lacking. A state of immunosuppression induced by sepsis has been demonstrated in clinical and experimental sepsis (3), with the majority of patients who have succumbed to sepsis-associated mortality showing evidence of immunosuppression and unresolved septic foci (4).

Polymorphonuclear neutrophils (PMNs) are an essential component of the innate immune system, involved in the clearance of extracellular pathogens (5). As the most abundant subset of leukocytes, the involvement of PMN in sepsis is significant $(3,6,7)$. The migration of PMNs is regulated by chemoattractants and chemokine gradients $(3,7,8)$. CXC-chemokine receptor (CXCR) 1 and CXCR2 are the major chemokine receptors on PMNs, with interleukin (IL)-8 acting as a ligand of these receptors (9). Severe sepsis is associated with the failure of PMNs to migrate (10). In a previous clinical study, patients with suppression of PMN receptors were predisposed to inflammatory response syndrome (11). Previous in vitro investigations have demonstrated that CXCR2 is downregulated upon stimulation with tumor necrosis factor- $\alpha$ (TNF- $\alpha)(12)$. The activation of Toll-like receptor 4 (TLR4) also suppresses the expression of CXCR2 (13). In an experimental mouse model of sepsis, the failure of PMNs to migrate was shown to result in a high rate of mortality (1). Tancevski et al and Van Zee et al reported that promoting the recruitment of PMNs ameliorates sepsis and attenuates sepsis-related injury and infection, respectively $(14,15)$. Therefore, in order to improve treatment of inflammatory disorders, including sepsis, the promotion of PMN chemotaxis is an attractive target (6). 
As an ancient Chinese herb, Folium isatidis is considered to have detoxification properties in traditional Chinese medicine. In addition to being used to cure febrile diseases, including dipsosis, fever, jaundice and hematemesis, F. isatidis is also deployed against diseases, including mumps, viral hepatitis, influenza and epidemic encephalitis B (16-18). It was reported to be effective during the 2003 severe acute respiratory syndrome flu outbreak (19). These antimicrobial and anti-endotoxic properties indicate its potential to be developed into a natural antibiotic $(20,21)$. In our previous study (22), the main chemical components of $F$. isatidis were identified using high-performance liquid chromatography. It was also demonstrated that $F$. isatidis increased the survival of septic mice, and ameliorated lung injury by inhibiting the production of inflammatory cytokines TNF- $\alpha$ and IL- 6 through the myeloid differentiation primary response gene 88 and nuclear factor $-\kappa B$ pathways (22). Although the beneficial immunomodulatory effects of $F$. isatidis have been the focus of previous investigations, whether this herb has an effect on chemokine receptors remains to be elucidated. The present study aimed to determine whether F. isatidis affects the migration of PMNs.

\section{Materials and methods}

Isolation of neutrophils. The present study was approved by the Ethics Committee of The Second Hospital of Wenzhou Medical University (Wenzhou, China), and informed consent was obtained. Peripheral blood was collected from four 20-30-year-old female healthy volunteers between August and December 2015, who had been referred to The Second Affiliated Hospital and Yuying Children's Hospital of Wenzhou Medical University and who provided informed, written consent. The blood was transferred into heparin lithium-containing tubes (BD Bioscience, San Jose, CA, USA). The samples were processed within $1 \mathrm{~h}$ of collection. Whole blood was incubated with 3\% dextran T-500 in the dark at room temperature for 20 min for sedimentation of red blood cells (RBCs). Following sedimentation, the white blood cell-enriched upper phase was layered over a Ficoll-Paque solution (GE Healthcare Life Science, Uppsala, Sweden). Centrifugation was performed at $427 \mathrm{x} \mathrm{g}$ at room temperature for $20 \mathrm{~min}$ without breaks, resulting in four distinct layers. The first three layers were discarded, and the remaining layer containing granulocytes and remnant RBCs was diluted in ACK lysis buffer (Gibco; Thermo Fisher Scientific, Inc., Waltham, MA, USA). The cells were then washed with PBS, and the freshly isolated neutrophils were resuspended in RPMI 1640 (Gibco; Thermo Fisher Scientific, Inc.). The purity of the isolated neutrophils was $>90 \%$ based on Wright's staining and differential counts.

Flow cytometry. The isolated neutrophils were diluted to $1 \times 10^{6}$ cells/ml with RPMI 1640 (Gibco; Thermo Fisher Scientific, Inc.). The cells were then incubated with either vehicle $(0.01 \%$ DMSO) or increasing concentrations of $n$-butanol extract from Folium isatidis $(100,250$ and $500 \mu \mathrm{g} / \mathrm{ml})$ at $37^{\circ} \mathrm{C}$ and $5 \% \mathrm{CO} 2$ for $2 \mathrm{~h}$. The specific method to obtain $n$-butanol extract from F. isatidis was described in previous study (22). The cells were then stimulated with $0.5 \mu \mathrm{g} / \mathrm{ml}$ lipopolysaccharide (LPS; Sigma; Merck Millipore, Darmstadt, Germany) for $4 \mathrm{~h}$ at $37^{\circ} \mathrm{C}$. The cells were washed with ice-cold PBS and then resuspended in $50 \mu \mathrm{l}$ FACS buffer. The cells were then incubated with a 1:50 dilution of the following antibodies (the concentrations suggested by the manufacturer) for $30 \mathrm{~min}$ at $4^{\circ} \mathrm{C}$ in the dark: FITC-conjugated anti-human CD181 (CXCR1; cat. no 11-1819-42), PE-conjugated anti-human CD182 (CXCR2; cat. no 12-1829-42) and APC-conjugated anti-human CD11b (cat no. 17-0118-42) or FITC-conjugated anti-human L-selectin (CD62L; cat. no 11-0629-42), PE-conjugated anti-human TLR4 (cat no. 12-9917-41) and APC conjugated anti-human TLR2 (cat no. 17-9922-42) (all from eBioscience, San Diego, CA, USA). Antibodies of the same isotype were used as negative controls. The cells were analyzed using a FACS calibur system (BD Biosciences). The mean fluorescence intensity (MFI) for 10,000 cells in each sample was determined using CellQuest software, version 5.2 (BD Biosciences).

Chemotaxis assays. The isolated neutrophils were pretreated as described above for $2 \mathrm{~h}$ and then stimulated with LPS $(0.5 \mu \mathrm{g} / \mathrm{ml})$ for $4 \mathrm{~h}$. The cells were then washed twice with PBS, and resuspended in RPMI 1640 at $1 \times 10^{6}$ cells $/ \mathrm{ml}$. The chemotaxis assays were performed using Transwell inserts ( 24 wells, $5 \mu \mathrm{m}$ pore size; Corning, Inc., Corning, NY, USA), where 10,000 cells in $100 \mu \mathrm{l}$ were loaded in the upper inserts and $600 \mu \mathrm{l}$ of IL-8 (100 ng/ml; eBioscience; Thermo Fisher Scientific, Inc.) containing RPMI 1640 was added to the lower wells. Following co-incubation for $2 \mathrm{~h}$ at $37^{\circ} \mathrm{C}$ and a humidified $5 \% \mathrm{CO}_{2}$ atmosphere, the inserts were removed, and the migrated neutrophils in the lower wells were collected and quantified according to myeloperoxidase (MPO) activity using a myeloperoxidase assay kit (Nanjing Jiancheng Bioengineering Institute, Nanjing, China).

Reverse transcription-quantitative polymerase chain reaction $(R T-q P C R)$ analysis. Total RNA was isolated from cells using TRIzol reagent (Invitrogen; Thermo Fisher Scientific, Inc.). RT-qPCR analysis was performed using an M-MLV Platinum SYBR Green qPCR SuperMix-UDG kit (Invitrogen; Thermo Fisher Scientific, Inc.) according to the manufacturer's protocol. A total of $1 \mu \mathrm{g}$ RNA was used for the determination. qPCR was performed in a $10 \mu \mathrm{l}$ reaction volume containing $2 \mu \mathrm{l}$ cDNA target, $2.25 \mu \mathrm{l} \mathrm{SYBR}$ Green (Bio-Rad Laboratories, Inc., Hercules, CA, USA), $0.25 \mu 1$

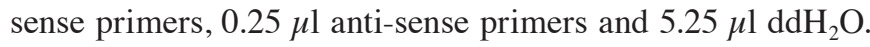
An Eppendorf Mastercycler realplex detection system (Eppendorf, Hamburg, Germany) was used for RT-qPCR analysis: The reaction consisted of the following conditions: $95^{\circ} \mathrm{C}$ for $3 \mathrm{~min}$, followed by $95^{\circ} \mathrm{C}$ for $15 \mathrm{sec}, 60^{\circ} \mathrm{C}$ for $30 \mathrm{sec}$, and $70^{\circ} \mathrm{C}$ for $30 \mathrm{sec}$ ( 40 cycles). The primers of the genes were synthesized by Invitrogen (Thermo Fisher Scientific, Inc.) and the sequences were as follows: Human CXCR1, sense 5'-GCA GCTCCTACTGTTGGACA-3' and antisense 5'-GGGCAT AGGCGATGATCACA-3'; human CXCR2, sense 5'-CCC ATCTTCATTCTTCGGAC-3', and antisense 5'-GACAAT GTTGTAGGGAAGCCAG-3'; human TLR2, sense 5'-ATG CCTACTGGGTGGAGAAC-3' and antisense 5'-TGCACC ACTCAGTCTTCACA-3'; human TLR4, sense 5'-CAGCTC TTGGTGGAAGTTGA-3' and antisense 5'-GCAAGAAGC ATCAGGTGAAA-3'; human CD62L, sense 5'-CTCCTTGCC AGCCAAATGATAA-3' and antisense 5'-CCTCTTCATTCC AGTGGCAGTC-3'; human CD11b, sense 5'-GGACCTCGG GCTCAAGTAAT-3' and antisense 5'-GCCTGTAATGCC 
AGCACTTT-3'; human IL-8, sense 5'-CTGGCCGTGGCT CTCTTG-3' and antisense 5'-CCTTGGCAAAACTGCACC TT-3'; human $\beta$-actin, sense 5'-CCTGGCACCCAGCACAAT' and antisense 5'-GCCGATCCACACGGAGTACT-3'. The result of real-time PCR was expressed as the threshold cycle (CT). The CT represents the PCR cycle at which the reported fluorescence rises above a set baseline threshold when the DNA amplicon is replicating exponentially (12). Results were normalized to $\beta$-actin as plotted as relative expression to the average of CON or DMSO, which were set as 100.

Quantification of $I L-8$. The neutrophils were pretreated for $2 \mathrm{~h}$ with the extract as described above, and then stimulated with LPS $(0.5 \mu \mathrm{g} / \mathrm{ml})$ for 1 or $4 \mathrm{~h}$. The supernatant and cells were collected, and the levels of IL-8 in the supernatant were determined using enzyme-linked immunosorbent assay (ELISA) kits (cat no. 88-8086-88; eBioscience; Thermo Fisher Scientific, Inc.) according to the manufacturer's protocol. The total quantity of IL-8 in the supernatant was normalized to that of total protein in the viable cell pellets.

Statistical analysis. All the experiments were performed in triplicate. Data are expressed as the mean \pm standard error of the mean. All the data were statistically analyzed using Student's $t$ test. $\mathrm{P}<0.05$ was considered to indicate a statistically significant difference. GraphPad Prism 5.0 software (GraphPad Software, Inc., La Jolla, CA, USA) was used for statistical analysis.

\section{Results}

LPS downregulates neutrophil expression of CXCR1, CXCR2 and CD62L. TLR2 and TLR4/myeloid differentiation factor 2 are vital in the recognition of LPS in the host, and the triggering of these receptors can lead to neutrophil recruitment and migration via CXCR1 and CXCR2. CD11b and CD62L, which are expressed on the surface of neutrophils, are critical for the occurrence of sepsis. LPS, also known as endotoxin, is a component of the outer membrane of Gram-negative bacteria, and is known to induce septic shock when present in high quantities. Therefore, the present study measured the expression of neutrophil chemokine receptors under LPS stimulation. The isolated neutrophils were treated with either vehicle or increasing concentrations $(10,100$ and $1,000 \mathrm{ng} / \mathrm{ml})$ of LPS for $4 \mathrm{~h}$, and the expression levels of CXCR1, CXCR2, TLR2, TLR4, CD11b and CD62L were measured using flow cytometry. The results showed that LPS treatment resulted in decreased expression levels of CXCR1, CXCR2 and CD62L in a dose-dependent manner (Fig. 1A-C), which was in accordance with the results of a previous study (23). However, no significant changes were observed in the expression levels of TLR2, TLR4 or CD11b in the LPS-treated neutrophils, compared with the vehicle-treated neutrophils (Fig. 1D-F). Therefore, the expression levels of TLR2, TLR4 and CD11b were not examined in the remainder of the experiments.

n-butanol extract from Folium isatidis prevents the LPS-induced downregulation of CXCR1, CXCR2 and CD62L. CXCR1, CXCR2 and CD62L may be important in sepsis, however, whether $n$-butanol extract affects these chemokine receptors remains to be elucidated. Therefore, the present study assessed the effect of the extract on the expression of CXCR1, CXCR2 and CD62L. The isolated neutrophils were pre-incubated with either vehicle or increasing concentrations of extract $(100,250$ and $500 \mu \mathrm{g} / \mathrm{ml})$ for $2 \mathrm{~h}$, and were subsequently treated with LPS $(0.5 \mu \mathrm{g} / \mathrm{ml})$ for $4 \mathrm{~h}$. Subsequent analysis of the cells using flow cytometry indicated that the extract inhibited the LPS-induced downregulation of CXCR1, CXCR2 and CD62L (Fig. 2A-C) in a dose-dependent manner.

Effect of n-butanol extract from Folium isatidis on the gene expression levels of CXCR1, CXCR2 and CD62L. As the $n$-butanol extract obtained from $F$. isatidis had a significant effect on chemokine receptor protein levels, the present study investigated the gene expression levels of these receptors. The isolated neutrophils were pretreated with extract and then treated with LPS $(0.5 \mu \mathrm{g} / \mathrm{ml})$ for 1 or $4 \mathrm{~h}$ (Fig. 3A-F). The extract increased the gene expression levels of CXCR1, CXCR2 and CD62L in a dose-dependent manner.

n-butanol extract from Folium isatidis decreases the expression of $I L-8$ and increases the activity of MPO. IL-8, as the ligand of CXCR1 and CXCR2, is important in the regulation of neutrophil migration. Following exposure of the neutrophils to LPS for $4 \mathrm{~h}$, the mRNA expression of IL-8 was significantly increased (Fig. 4A). Therefore, the present study examined whether $n$-butanol extract affected the production of IL-8. The isolated neutrophils were pretreated with extract and then incubated with LPS $(0.5 \mu \mathrm{g} / \mathrm{ml})$ for $1 \mathrm{~h}$ (Fig. 4B) or $4 \mathrm{~h}$ (Fig. 4C). The expression levels of IL-8 were quantified using ELISA. A decrease in IL-8 was observed following exposure to LPS for 1 and $4 \mathrm{~h}$ when pretreated with the extract. Specifically, following exposure to LPS for $4 \mathrm{~h}$, the cytokine expression of IL- 8 was decreased $>5$-fold when the cells were treated with the extract at a dose of $500 \mu \mathrm{g} / \mathrm{ml}$, compared with LPS stimulation (Fig. 4C). MPO levels are a measure of neutrophil migration. Therefore, the present study examined the effect of the extract on the activity of MPO. Neutrophils were pretreated with $n$-butanol extract for $2 \mathrm{~h}$ followed by stimulation with LPS for $4 \mathrm{~h}$. The activity of MPO increased in a dose-dependent manner following treatment with extract, compared with the control (Fig. 4D), suggesting that $n$-butanol extract promoted the function of neutrophils.

\section{Discussion}

Sepsis remains a threat to human health, primarily due to a lack of effective therapies (3). With current treatment options, the mortality rate of patients with sepsis remains high at $\sim 30 \%$, and mortality rates increase directly with disease severity (24). A previous worldwide survey of relevant pathogens in intensive care units found that the majority of patients with sepsis had blood cultures positive for Gram-negative bacteria (25). LPS, a component of the outer leaflet of the outer membrane of Gram-negative bacteria, is a key molecule in the induction of sepsis. Therefore, the present study used LPS to simulate sepsis induced by Gram-negative bacteria infection.

As previously reported, the present study observed that LPS stimulation decreased the expression of CXCR1, CXCR2 and CD62L on neutrophils. In addition to being effector cells 
A

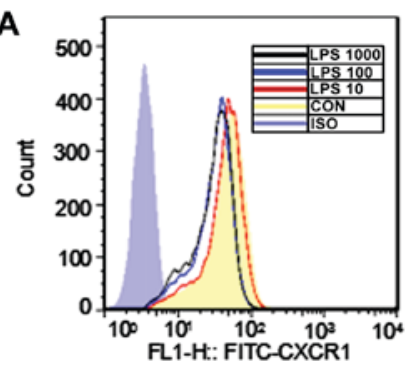

CXCR1

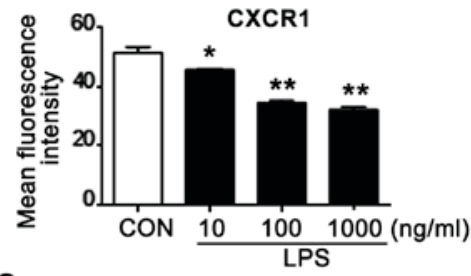

C

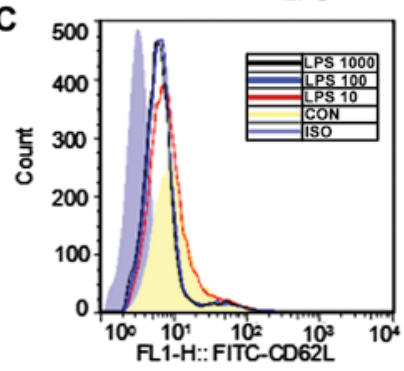

CD62L

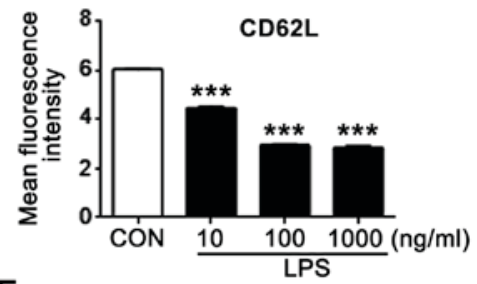

E

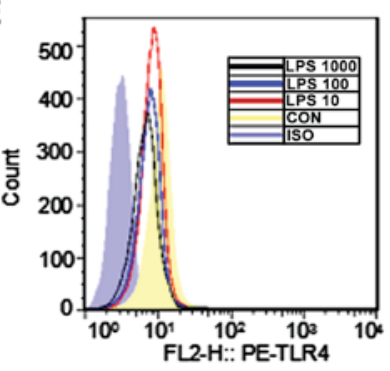

TLR4

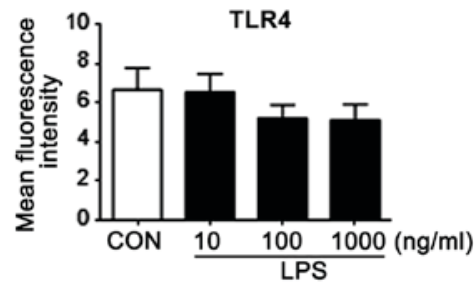

B
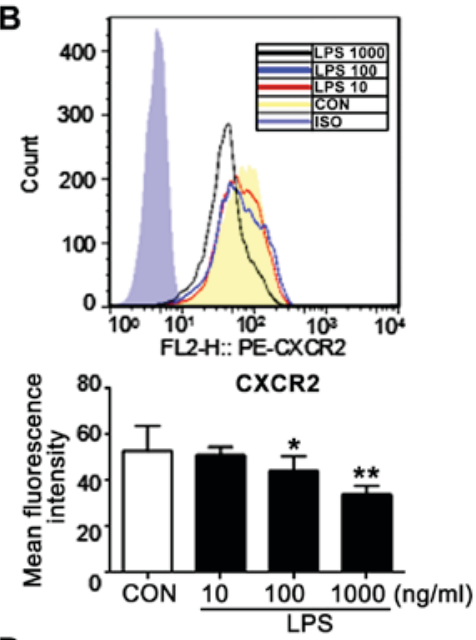

D
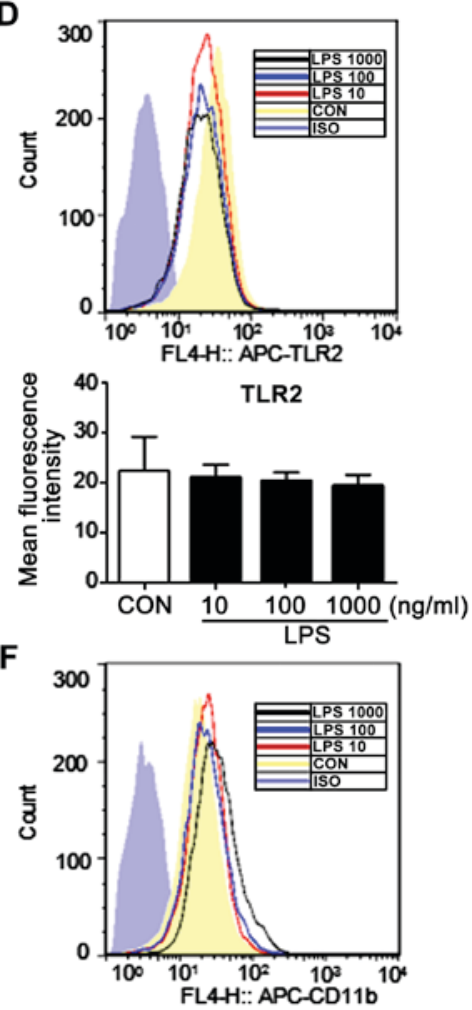

CD11b

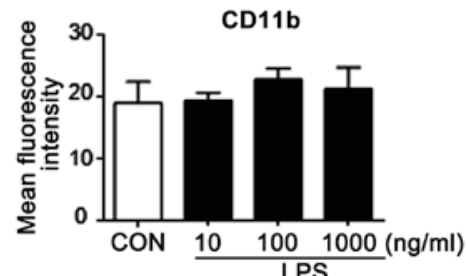

Figure 1. LPS induces a decrease in the neutrophilic expression of CXCR1, CXCR2 and CD62L. Isolated neutrophils were incubated with LPS for $4 \mathrm{~h}$. Levels of (A) CXCR1, (B) CXCR2, (C) CD62L, (D) TLR2, (E) TLR4 and (F) CD11b were measured using flow cytometry. The corresponding mean fluorescence intensity was calculated. Each bar represents the mean \pm standard error of the mean of three independent experiments. Statistical significance relative to the CON group was determined. ${ }^{*} \mathrm{P}<0.05,{ }^{* *} \mathrm{P}<0.01$ and ${ }^{* * *} \mathrm{P}<0.001$. LPS, lipopolysaccharide; CXCR1, CXC-chemokine receptor 1; CXCR2, CXC-chemokine receptor 2; CD62L, L-selectin; TLR2, toll-like receptor 2; TLR4, toll-like receptor 4; CON, control.

involved in the acute phase of the inflammatory response, neutrophils are also important in the resolution of inflammation. The failure of neutrophils to migrate can lead to an inability to control infection due to weakened neutrophil phagocytic and bactericidal abilities (26). CXCR1 and CXCR2 are the major chemokine receptors on neutrophils. Chemokine receptors regulate the migration of neutrophils to the site of infection to assist in controlling invading pathogens and protecting the body. Duerschmied et al (27) demonstrated that survival following LPS-induced endotoxic shock improved upon the promotion of neutrophil recruitment during acute inflammation. $n$-butanol extract was found to have significant antiseptic abilities in our previous study. However, whether the extract affected neutrophil migration remained unclear. In 
A

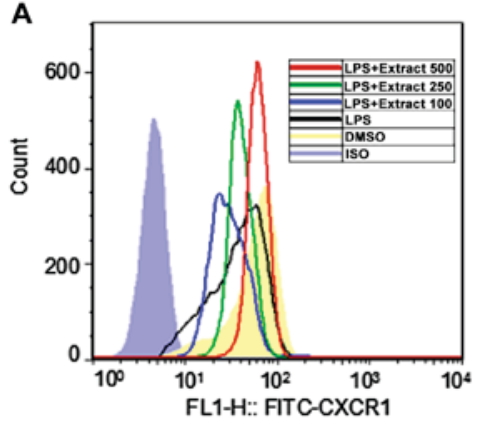

B
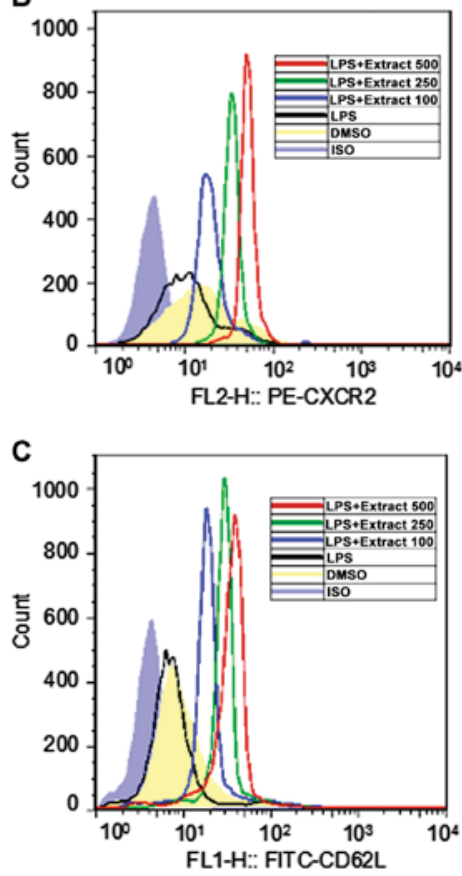

CXCR1

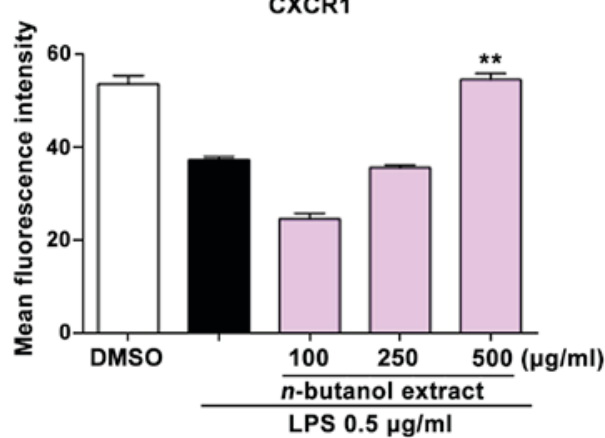

CXCR2

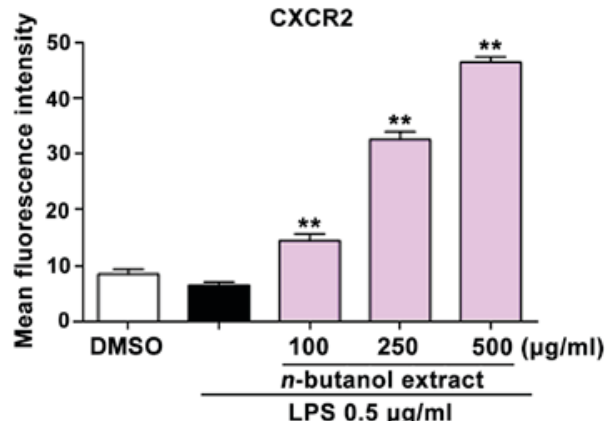

CD62L

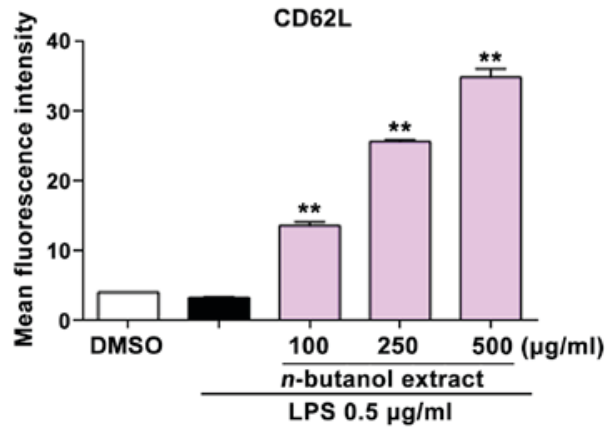

Figure 2. $n$-butanol extract from Folium isatidis inhibits the downregulated protein levels of CXCR1, CXCR2 and CD62L. Following pretreatment with a vehicle control (DMSO) or $n$-butanol extract at the indicated concentration for $2 \mathrm{~h}$, neutrophils were incubated with LPS ( $0.5 \mu \mathrm{g} / \mathrm{ml})$ for $4 \mathrm{~h}$. Expression levels of (A) CXCR1, (B) CXCR2 and (C) CD62L were measured using flow cytometry. The corresponding mean fluorescence intensity was calculated. Each bar represents the mean \pm standard error of the mean of three independent experiments. Statistical significance relative to the LPS group was determined. ${ }^{* *} \mathrm{P}<0.01$. LPS, lipopolysaccharide; CXCR1, CXC-chemokine receptor 1; CXCR2, CXC-chemokine receptor 2; CD62L, L-selectin.

the present study, it was demonstrated that $n$-butanol extract prevented the downregulation of CXCR1, CXCR2 and CD62L.

In our previous study, it was demonstrated that $n$-butanol extract from Folium isatidis significantly inhibited the activation of TLR4 and its signaling pathways (22). It has been reported that the systemic activation of TLRs and high levels of TNF- $\alpha$ are involved in the reduction of neutrophil recruitment through the downregulation of CXCR2 in neutrophils (28). Therefore, it was hypothesized that $n$-butanol extract from $F$. isatidis prevents the downregulation of CXCR1 and CXCR2 through the activation of TLR4 and secretion of TNF- $\alpha$.

The primary function of CD62L, a vascular adhesion molecule, is directing neutrophil migration. In addition to CXCR1 and CXCR2, neutrophil migration requires the assistance of CD62L. In the present study, the expression of CD62L decreased following incubation with LPS for $4 \mathrm{~h}$ (Fig. 1C), whereas $n$-butanol extract promoted the expression of CD62L in a dose-dependent manner (Fig. 2C). This suggested that the extract promoted the migration of neutrophils by inhibiting the sepsis-induced downregulation of CD62L.
IL- 8 is a ligand of CXCR1 and CXCR2, and is rapidly secreted upon cell stimulation. The mRNA expression of IL-8 significantly increased upon stimulation with LPS (Fig. 4A). However, $n$-butanol extract decreased the expression of IL- 8 in a dose-dependent manner (Fig. 4B and C), and prevented the decreases in the expression of CXCR1 and CXCR2. This suggested that the extract increased the activity of neutrophils and upregulated the expression of chemokine receptors, including CXCR1 and CXCR2, rendering neutrophils more sensitive to the ligand IL-8. The present study quantified MPO activity as a measure of neutrophil migration. It was found the extract promoted the activity of MPO. The extract also promoted the ability of neutrophils to migrate by increasing the expression of CXCR1 and CXCR2 on the surface of neutrophils.

To date, the treatment of sepsis consists primarily of supportive measures and experimental therapeutic approaches (29). Therefore, novel pharmacological strategies are urgently required to promote the treatment of sepsis (30). With the ability to promote the expression of CXCR1, CXCR2 

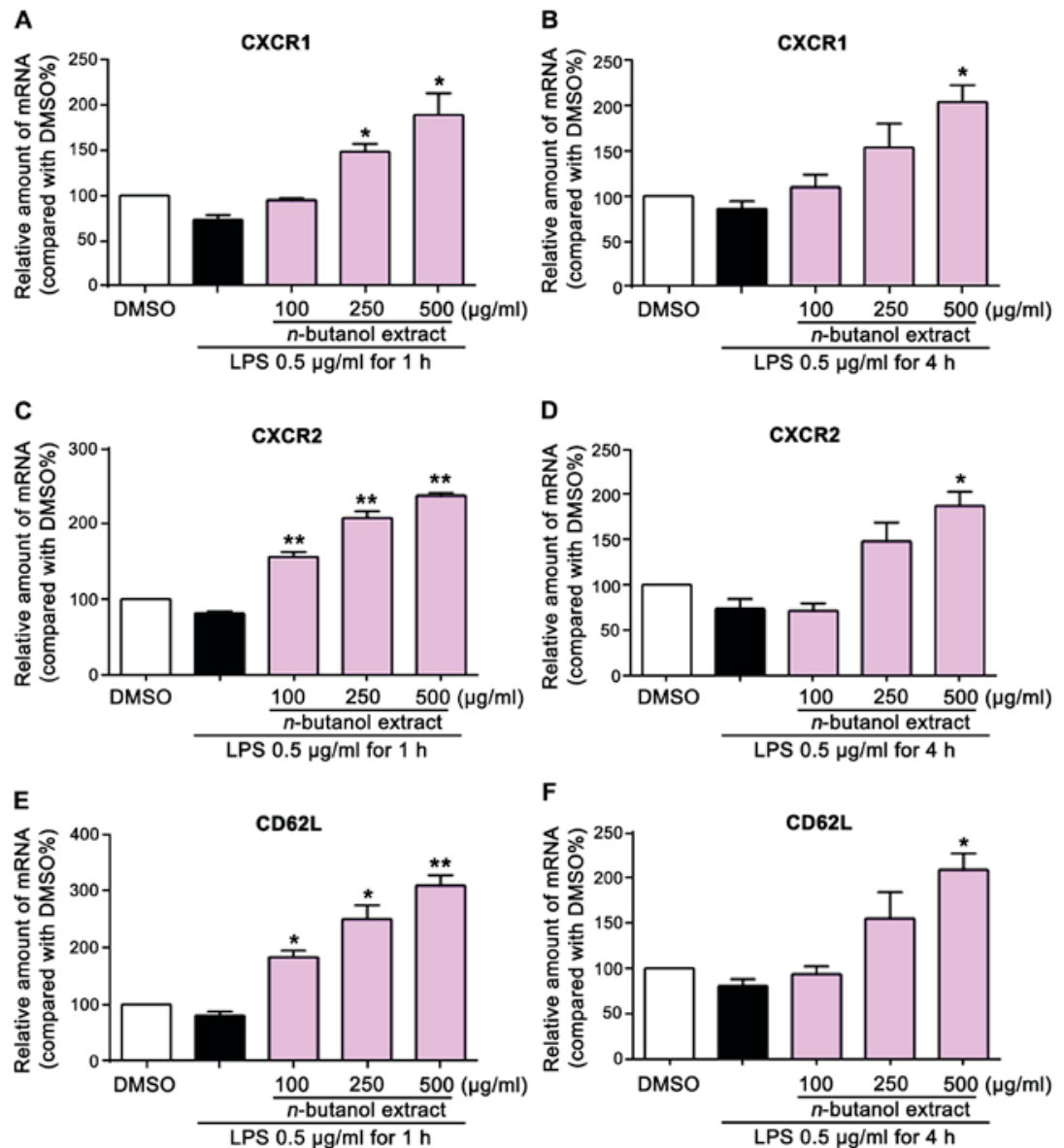

Figure 3. Treatment with $n$-butanol extract from Folium isatidis inhibits downregulation in the gene levels of CXCR1, CXCR2 and CD62L. Neutrophils were pretreated with a vehicle control (DMSO) or the indicated concentration of $n$-butanol extract for $2 \mathrm{~h}$. The neutrophils were then incubated with LPS ( $0.5 \mu \mathrm{g} / \mathrm{ml})$ for 1 or $4 \mathrm{~h}$. The mRNA levels of CXCR1 at (A) $1 \mathrm{~h}$ and (B) $4 \mathrm{~h}$; CXCR2 at (C) $1 \mathrm{~h}$ and (D) $4 \mathrm{~h}$; and CD62L at (E) $1 \mathrm{~h}$ and (F) $4 \mathrm{~h}$ were determined using RT-qPCR analysis. The results are presented as the percentage of the control. Each bar represents the mean \pm standard error of the mean of three independent experiments. Statistical significance relative to the LPS group was determined. " $\mathrm{P}<0.05$; ${ }^{* *} \mathrm{P}<0.01$. LPS, lipopolysaccharide; CXCR1, CXC-chemokine receptor 1; CXCR2, CXC-chemokine receptor 2; CD62L, L-selectin; RT-qPCR, reverse transcription-quantitative polymerase chain reaction.
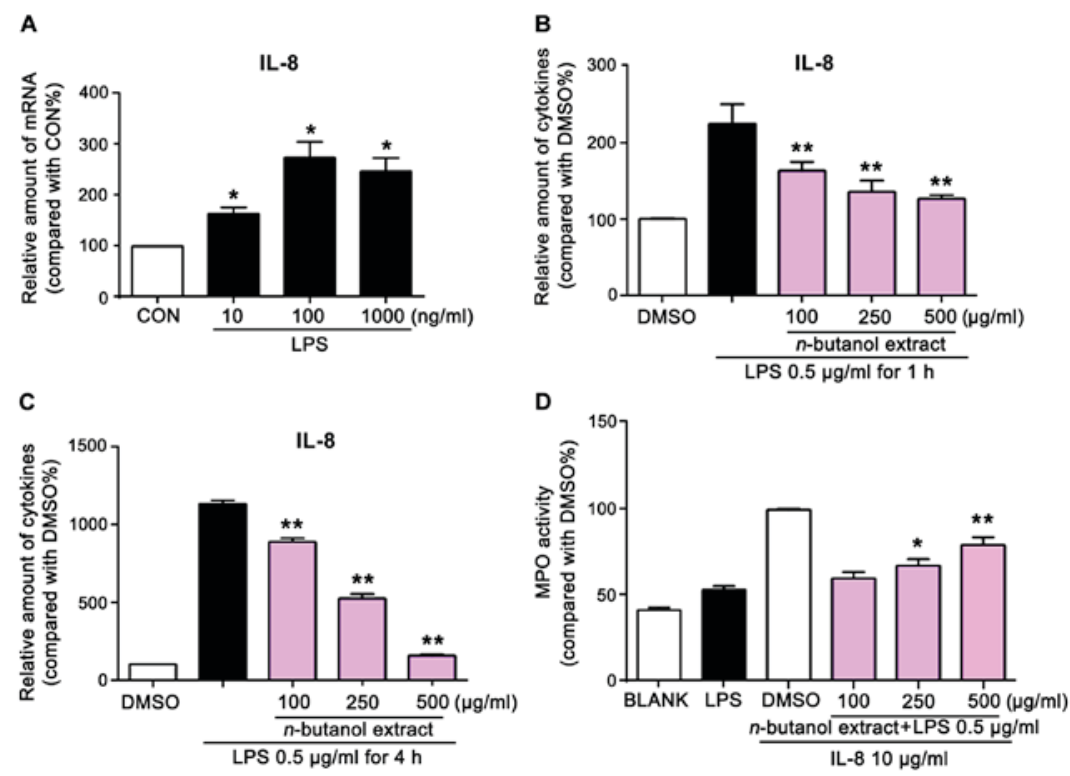

Figure 4. $n$-butanol extract from Folium isatidis inhibits the expression of IL-8 and enhances neutrophil MPO activity. (A) Gene expression levels of IL-8 were examined using reverse transcription-quantitative polymerase chain reaction analysis following exposure of isolated neutrophils to LPS for $4 \mathrm{~h}$. Following pretreatment with a vehicle control (DMSO) or the indicated concentrations of $n$-butanol extract for $2 \mathrm{~h}$, neutrophils were incubated with LPS (0.5 $\mu \mathrm{g} / \mathrm{ml})$ for (B) $1 \mathrm{~h}$ or (C) $4 \mathrm{~h}$. The protein levels of IL-8 were measured using an enzyme-linked immunosorbent assay. (D) MPO activity was examined using a chemotaxis assay. The results are presented as a percentage of the control. Each bar represents the mean \pm standard error of the mean of three independent experiments. Statistical significance relative to the LPS group was determined. ${ }^{*} \mathrm{P}<0.05 ;{ }^{* * *} \mathrm{P}<0.01$. MPO, myeloperoxidase; IL-8 interleukin-8; LPS, lipopolysaccharide. 
and CD62L, $n$-butanol extract itself is a potential candidate for the treatment of LPS-induced sepsis. However, due to the presence of multiple bioactive components in the extract and limited approaches for preparing extracts from $F$. isatidis, it is difficult to identify the precise agent inhibiting LPS-induced chemokine receptor downregulation. Although the main active chemical components have been identified, there are more than eight major compounds in $n$-butanol extract (22). Further investigation is required to identify the active compound in $F$. isatidis.

In conclusion, the present study demonstrated that $n$-butanol extract obtained from $F$. isatidis prevented the LPS-induced downregulation of CXCR1, CXCR2 and CD62L on neutrophils. The extract promoted neutrophil migration in response to IL-8. These results indicated that $n$-butanol extract from $F$. isatidis, which targets neutrophil chemotaxis during LPS-induced sepsis, is a potential candidate for the treatment of sepsis.

\section{Acknowledgements}

This study was supported by the Provincial Natural Science Foundation of Zhejiang (grant no. Y2100530).

\section{References}

1. Lee SK, Kim SD, Kook M, Lee HY, Ghim J, Choi Y, Zabel BA, Ryu SH and Bae YS: Phospholipase D2 drives mortality in sepsis by inhibiting neutrophil extracellular trap formation and down-regulating CXCR2. J Exp Med 212: 1381-1390, 2015.

2. Prucha M, Bellingan G and Zazula R: Sepsis biomarkers. Clin Chim Acta 440: 97-103, 2015.

3. Drifte G, Dunn-Siegrist I, Tissières $P$ and Pugin J: Innate immune functions of immature neutrophils in patients with sepsis and severe systemic inflammatory response syndrome. Crit Care Med 41: 820-832, 2013.

4. Silva SC, Baggio-Zappia GL, Brunialti MK, Assunçao MS, Azevedo LC, Machado FR and Salomao R: Evaluation of Toll-like, chemokine, and integrin receptors on monocytes and neutrophils from peripheral blood of septic patients and their correlation with clinical outcomes. Braz J Med Biol Res 47: 384-393, 2014.

5. Seeley EJ, Matthay MA and Wolters PJ: Inflection points in sepsis biology: From local defense to systemic organ injury. Am J Physiol Lung Cell Mol Physiol 303: L355-L363, 2012.

6. Boppana NB, Devarajan A, Gopal K, Barathan M, Bakar SA, Shankar EM, Ebrahim AS and Farooq SM: Blockade of CXCR2 signalling: A potential therapeutic target for preventing neutrophil-mediated inflammatory diseases. Exp Biol Med (Maywood) 239: 509-518, 2014.

7. Jaillon S, Galdiero MR, Del Prete D, Cassatella MA, Garlanda C and Mantovani A: Neutrophils in innate and adaptive immunity. Semin Immunopathol 35: 377-394, 2013.

8. Park DW, Jiang S, Tadie JM, Stigler WS, Gao Y, Deshane J, Abraham E and Zmijewski JW: Activation of AMPK enhances neutrophil chemotaxis and bacterial killing. Mol Med 19: 387-398, 2013

9. Takahashi M, Ishiko T, Kamohara H, Hidaka H, Ikeda O, Ogawa $\mathrm{M}$ and Baba H: Curcumin (1,7-bis(4-hydroxy-3-metho xyphenyl)-1,6-heptadiene-3,5-dione) blocks the chemotaxis of neutrophils by inhibiting signal transduction through IL-8 receptors. Mediators Inflamm 2007: 10767, 2007.

10. Ishii M, Asano K, Namkoong H, Tasaka S, Mizoguchi K, Asami T, Kamata H, Kimizuka Y, Fujiwara H, Funatsu Y, et al: CRTH2 is a critical regulator of neutrophil migration and resistance to polymicrobial sepsis. J Immunol 188: 5655-5664, 2012.

11. Tarlowe MH, Duffy A, Kannan KB, Itagaki K, Lavery RF, Livingston DH, Bankey P and Hauser CJ: Prospective study of neutrophil chemokine responses in trauma patients at risk for pneumonia. Am J Respir Crit Care Med 171: 753-759, 2005.

12. Tikhonov I, Doroshenko T, Chaly Y, Smolnikova V, Pauza CD and Voitenok N: Down-regulation of CXCR1 and CXCR2 expression on human neutrophils upon activation of whole blood by S. aureus is mediated by TNF-alpha. Clin Exp Immunol 125 : 414-422, 2001.
13. Deng M, Ma T, Yan Z, Zettel KR, Scott MJ, Liao $H$, Frank A, Morelli AE, Sodhi CP, Hackam DJ and Billiar TR: Toll-like receptor 4 signaling on dendritic cells suppresses polymorphonuclear leukocy te CXCR 2 expression and trafficking via interleukin 10 during intra-abdominal sepsis. J Infect Dis 213: 1280-1288, 2016.

14. Tancevski I, Nairz M, Duwensee K, Auer K, Schroll A, Heim C, Feistritzer C, Hoefer J, Gerner RR, Moschen AR, et al: Fibrates ameliorate the course of bacterial sepsis by promoting neutrophil recruitment via CXCR2. EMBO Mol Med 6: 810-820, 2014.

15. Van Zee KJ, DeForge LE, Fischer E, Marano MA, Kenney JS, Remick DG, Lowry SF and Moldawer LL: IL-8 in septic shock, endotoxemia, and after IL-1 administration. J Immunol 146: 3478-3482, 1991

16. Deng YP, Liu YY, Liu Z, Li J, Zhao LM, Xiao H, Ding XH and Yang ZQ: Antiviral activity of Folium isatidis derived extracts in vitro and in vivo. Am J Chin Med 41: 957-969, 2013.

17. Liu Z, Yang ZQ and Xiao H: Antiviral activity of the effective monomers from Folium Isatidis against influenza virus in vivo. Virol Sin 25: 445-451, 2010.

18. Fang JG, Hu Y, Tang J, Wang WQ and Yang ZQ: Antiviral effect of Folium Isatidis on herpes simplex virus type I. Zhongguo Zhong Yao Za Zhi 30: 1343-1346, 2005 (In Chinese).

19. Lau TF, Leung PC, Wong EL, Fong C, Cheng KF, Zhang SC, Lam CW, Wong V, Choy KM and Ko WM: Using herbal medicine as a means of prevention experience during the SARS crisis. Am J Chin Med 33: 345-356, 2005.

20. Liu CS, Cham TM, Yang CH, Chang HW, Chen $\mathrm{CH}$ and Chuang LY: Antibacterial properties of Chinese herbal medicines against nosocomial antibiotic resistant strains of Pseudomonas aeruginosa in Taiwan. Am J Chin Med 35: 1047-1060, 2007.

21. Liau BC, Jong TT, Lee MR and Chen SS: LC-APCI-MS method for detection and analysis of tryptanthrin, indigo and indirubin in daqingye and banlangen. J Pharm Biomed Anal 43: 346-351, 2007.

22. Jiang L, Lu Y, Jin J, Dong L, Xu F, Chen S, Wang Z, Liang $\mathrm{G}$ and Shan $\mathrm{X}$ : n-Butanol extract from Folium isatidis inhibits lipopolysaccharide-induced inflammatory cytokine production in macrophages and protects mice against lipopolysaccharide-induced endotoxic shock. Drug Des Devel Ther 9: 5601-5609, 2015

23. Khandaker MH, Xu L, Rahimpour R, Mitchell G, DeVries ME, Pickering JG, Singhal SK, Feldman RD and Kelvin DJ: CXCR1 and CXCR2 are rapidly down-modulated by bacterial endotoxin through a unique agonist-independent, tyrosine kinase-dependent mechanism. J Immunol 161: 1930-1938, 1998.

24. Iskander KN, Craciun FL, Stepien DM, Duffy ER, Kim J, Moitra R, Vaickus LJ, Osuchowski MF and Remick DG: Cecal ligation and puncture-induced murine sepsis does not cause lung injury. Crit Care Med 41: 159-170, 2013.

25. Vincent JL, Rello J, Marshall J, Silva E, Anzueto A, Martin CD, Moreno R, Lipman J, Gomersall C, Sakr Y, et al: International study of the prevalence and outcomes of infection in intensive care units. JAMA 302: 2323-2329, 2009.

26. Kipnis E: Neutrophils in sepsis: Battle of the bands. Crit Care Med 41: 925-926, 2013.

27. Duerschmied D, Suidan GL, Demers M, Herr N, Carbo C, Brill A, Cifuni SM, Mauler M, Cicko S, Bader M, et al: Platelet serotonin promotes the recruitment of neutrophils to sites of acute inflammation in mice. Blood 121: 1008-1015, 2013.

28. Sônego F, Alves-Filho JC and Cunha FQ: Targeting neutrophils in sepsis. Expert Rev Clin Immunol 10: 1019-1028, 2014.

29. Rivers E, Nguyen B, Havstad S, Ressler J, Muzzin A, Knoblich B, Peterson E and Tomlanovich M: Early Goal-Directed Therapy Collaborative Group: Early goal-directed therapy in the treatment of severe sepsis and septic shock. N Engl J Med 345: 1368-1377, 2001.

30. Czepielewski RS, Porto BN, Rizzo LB, Roesler R, Abujamra AL, Pinto LG, Schwartsmann G, Cunha Fde Q and Bonorino C: Gastrin-releasing peptide receptor (GRPR) mediates chemotaxis in neutrophils. Proc Natl Acad Sci USA 109: 547-552, 2012.

This work is licensed under a Creative Commons Attribution-NonCommercial-NoDerivatives 4.0 International (CC BY-NC-ND 4.0) License. 